Rapid Reviews COVID-19

\title{
Reviews of "Reopening universities during the COVID-19 pandemic: A testing strategy to minimize active cases and delay outbreaks"
}

Dominique Gibert ${ }^{1}$, Mohak Gupta ${ }^{2}$, Rishika Mohanta ${ }^{3}$, Arthur Reingold $^{4}$, Alalli Mériem ${ }^{5}$

${ }^{1}$ Lyon 1 University, ${ }^{2}$ All India Institute of Medical Sciences (AIIMS), New Delhi, ${ }^{3}$ Indian Institute of Science Education and Research (IISER), Pune, ${ }^{4}$ University of California, Berkeley, SPH, ${ }^{5}$ Critical care and Emergency unit (SAU-SAMU-SMUR)

Published on: Sep 02, 2020

DOI: $10.1162 / 2 \mathrm{e} 3983 f 5.25016895$

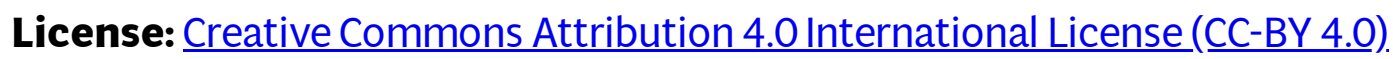


To read the original manuscript, click the link above.

Summary of Reviews: Pre-entry screening of students entering universities for COVID-19 may help limit the spread of COVID-19, but further analysis is warranted to know the true impact. The modeling is too simple for a complex situation, and should take into account other critical factors.

Reviewer 1 (Dominique Gibert)

Reviewer 2 (Mohak Gupta, Rishika Mohanta)

Reviewer 3 (Arthur Reingold) |

Reviewer 4 (Alalli Mériem)

RR:C19 Strength of Evidence Scale Key

प्रमप्र = Misleading

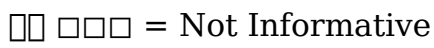

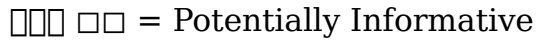

प्राप् = Reliable

प्राप्रा = Strong

To read the reviews, click the links below. 